\title{
QUASIFREE PHOTOPRODUCTION OF PION-PAIRS OF PROTONS AND NEUTRONS*
}

\author{
M. OBERLE ${ }^{\dagger}$ and B. KRUSCHE \\ (for the A2, Crystal Ball- and TAPS-collaborations) \\ Department of Physics, University of Basel, Klingelbergstrasse 82 \\ Ch-4056 Basel, Switzerland \\ ${ }^{\dagger}$ Markus.Oberle@unibas.ch
}

Published 14 January 2014

\begin{abstract}
Beam-helicity asymmetries and mass-differential cross sections have been measured at the MAMI accelerator in Mainz for the photoproduction of neutral and mixed-charge pion pairs in the reactions $\gamma p \rightarrow n \pi^{0} \pi^{+}$and $\gamma p \rightarrow p \pi^{0} \pi^{0}$ off free protons and $\boldsymbol{\gamma} d \rightarrow(p) p \pi^{0} \pi^{-}, \boldsymbol{\gamma} d \rightarrow(n) p \pi^{0} \pi^{0}$ and $\boldsymbol{\gamma} d \rightarrow(n) n \pi^{0} \pi^{+}, \boldsymbol{\gamma} d \rightarrow(p) n \pi^{0} \pi^{0}$ off quasi-free nucleons bound in the deuteron for incident photon energies up to $1.4 \mathrm{GeV}$. Circularly polarized photons were produced in bremsstrahlung processes of longitudinally polarized electrons and tagged with the Glasgow-Mainz magnetic spectrometer. The decay products (photons, protons neutrons and charged pions) were detected in the $4 \pi$ electromagnetic calorimeter composed of the Crystal Ball and TAPS detectors. Using a full kinematic reconstruction of the final state, excellent agreement was found between the results for free and quasi-free protons, indicate that the quasi-free neutron results are also a close approximation of the free-neutron results. Comparison of the results to predictions of model calculations portend that especially the reaction mechanisms in the production of the mixed-charge final states are still not well understood, in particular at low incident photon energies in the second nucleon resonance region.
\end{abstract}

\section{Introduction}

The fundamental properties of the strong interaction in the non-perturbative range are closely related to the excitation spectrum of nucleons, which is not fully understood yet and thus an intensivly discussed topic. The apparently poor match ${ }^{1}$ between the experimental database for excited nucleon states and model predictions based on Quantum Chromodynamics 'inspired' quark models has motivated huge efforts in experiment and also in theory development. To overcome the limitations in the available database, which was dominated by the results from pion scattering on nucleons and thus biased against nucleon resonances with small couplings to $N \pi$,

*This is an Open Access article published by World Scientific Publishing Company. It is distributed under the terms of the Creative Commons Attribution 3.0 (CC-BY) License. Further distribution of this work is permitted, provided the original work is properly cited. 
was the prime targed. Huge advances in accelerator and detector technology allow for photoproduction of mesons, which thus has become a prime tool in this research. Sequential decays involving intermediate excited states are playing an essential role in these processes and are especially important for resonances from the second and third resonance region, where the cross sections of many single-meson channels are already decreasing. These type of reactions have attracted much interest in recent years and thus the photoproduction of pseudoscalar mesons pairs, mostly pion pairs but also pion eta pairs, has been intensively experimentally studied ( $\mathrm{see}^{2-10}$ for recent results). The measurement of pion pairs with different charge combinations and also measurements of their production off both nucleons is mandatory for an isospin decomposition of the reaction, helping to identify contributions from $N^{\star}$ and $\Delta$ resonances. At least 23 independent variables one would have to measure to fix the amplitudes and phases in the description of photoproduction of pseudoscalar meson pairs ${ }^{11}$ and thus a fully complete measurement appears unrealistic. But already the measurement of at least some polarization observables can provide valuable constraints for the reaction models, since these observables are often very sensitiv to small details in the reaction mechanisms.

\section{Experiment and Analysis}

The experiments were performed at the tagged photon facility at the Mainz Microtron accelerator MAMI. ${ }^{12,13}$ Detailed information about the detector setup can be found in. ${ }^{14-21,4}$ The charged pions and the photons from the $\pi^{0}$ decay were detected in coincidence with the recoil nucleons. Standart analysis steps, like cuts on invariant mass $\mathrm{m}(\gamma \gamma)$, missing mass $\Delta M=\left|P_{\gamma}+P_{N}-P_{\pi^{0}}-P_{\pi^{ \pm}}\right|-m_{N}$ and coplanarity $\Delta \phi$ (azimuthal angular difference between the three-momenta of the two-pion system and the recoil nucleon), led to a very clean signal extraction, see Fig. 1.

The beam-helicity asymmetry is defined as: $I^{\odot}(\Phi)=\frac{1}{P_{\gamma}} \frac{d \sigma^{+}-d \sigma^{-}}{d \sigma^{+}+d \sigma^{-}}=\frac{1}{P_{\gamma}} \frac{N^{+}-N^{-}}{N^{+}+N^{-}}$ where $d \sigma^{ \pm}$are the differential cross sections for each of the two photon helicity states, and $P_{\gamma}$ is the degree of circular polarization of the photons (for detailed information on $\left.I^{\odot}(\Phi) \operatorname{see}^{23,24}\right)$.

A comparison of our free proton data to previously measured beam-helicity asymmetries $^{25}$ showed a reasonable agreement. In order to eliminate effects from the nuclear Fermi momentum in the quasi-free data a further analysis using the invariant mass of the final state, extracted from the four-momenta of the mesons and the recoil nucleons was performed and in an accurate comparison of the quasifree proton data to free proton data other nuclear effects could be excluded.

\section{Results and Conclusions}

The results for the quasi-free proton data show nice agreement with the free proton data demonstrating that the kinematic reconstruction of the final state reliably 

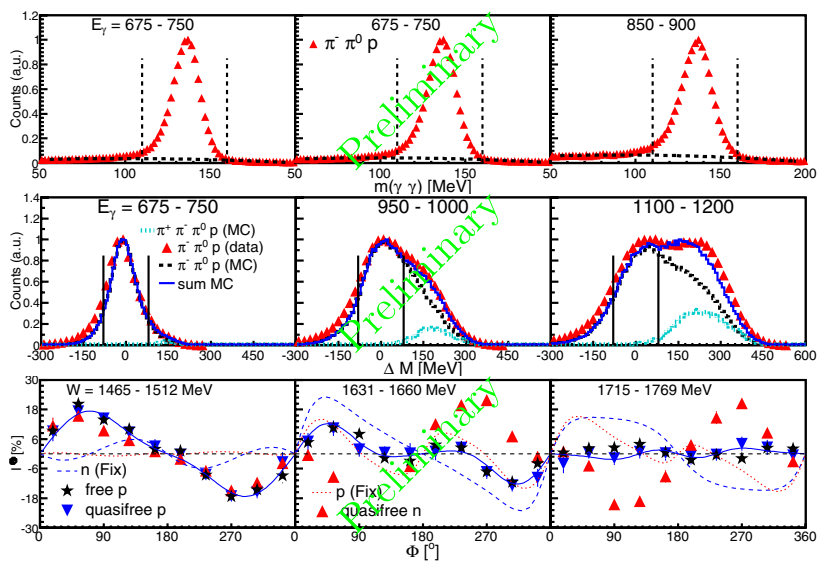

Fig. 1. Top and middle row: $m(\gamma \gamma)$ and $\Delta M$ for the $\pi^{-} \pi^{0} p$ final state, red triangles: data, vertical lines: applied cuts, dashed curve: background fit (only for $m(\gamma \gamma)$ ), black dashed line: MC for the $\pi^{-} \pi^{0} p$ final state, cyan short dashed line: MC of $\pi^{+} \pi^{-} \pi^{0}$ final states, blue full line sum of MC (only for $\Delta M$ ). bottom row: Beam helicity asymmetrie $I^{\odot}(\Phi)$ for the free (black stars) and quasi-free proton data (blue triangles) and for the quasi-free neutron data (red triangles) for the mixed-charge final states compared to model calculations from. ${ }^{22}$

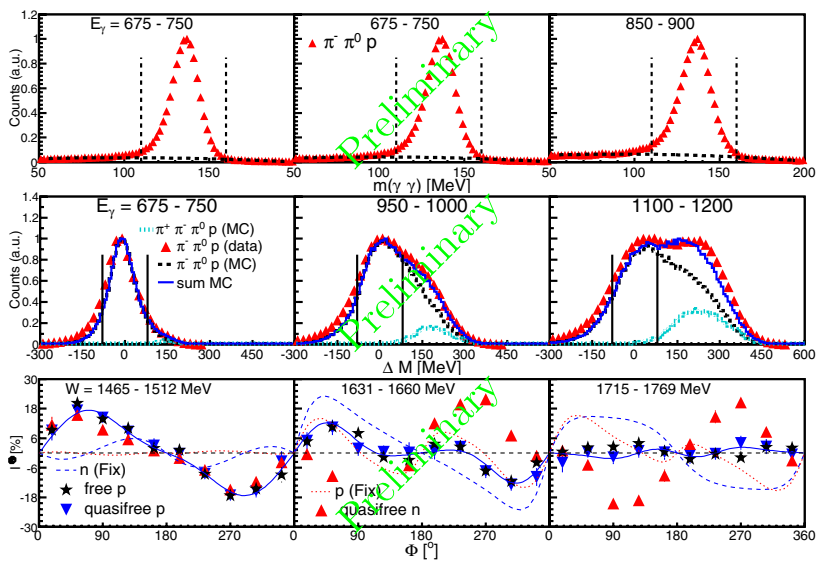

Fig. 2. left picture: coefficients of the fits to $I^{\odot}(\Phi)$ as function of the cm-energy $W$ for the neutral channel; left-hand side: free and quasi-free proton data, right-hand side: comparison of proton and neutron data. Solid curves: model results from. ${ }^{22}$ Dotted curves: solution BnGa2011-1 from, ${ }^{26}$ dash-dotted curves: solution BnGa2011-2 from. ${ }^{26}$ (Blue) diamonds: model results from. ${ }^{5}$

right picture: coefficients for the mixed-charge channel; same notation as above, model calculations from $^{22}$

eliminates effects from nuclear Fermi motion. This shows also that our quasi-free neutron data is a very good aproximation of a free neutron target.

Due to its symmetry properties, $I^{\odot}$ can be expanded in a sine series $\left(I^{\odot}(\Phi)=\right.$ $\left.\sum_{n=1}^{\infty} A_{n} \sin (n \Phi)\right)$ which can be fitted to the data. This allows for a more compact 
presentation of the results. In Fig. 2 the energy dependence of the four paramters $A_{n}$ are shown for free and quasi-free data and all four channels. The model claculations show a good agreement for $A_{1}$ of the neutral channel, whereas the agreement is less good for $A_{2}$. For the mixed-charge asymmetries the agreement is rather poor and it seems that over all these two-pion photoproduction mechanisms are still not very well understood.

This data should certainly help to strongly constrain future model analyses of this reactions.

\section{Acknowledgments}

We wish to acknowledge the outstanding support of the accelerator group and operators of MAMI. This work was supported by Schweizerischer Nationalfonds, Deutsche Forschungsgemeinschaft (SFB 443, SFB/TR 16), DFG-RFBR (Grant No. 05-02-04014), UK Science and Technology Facilities Council, STFC, European Community-Research Infrastructure Activity (FP6), the US DOE, US NSF and NSERC (Canada).

\section{References}

1. B. Krusche and S. Schadmand, Prog. Part. Nucl. Phys. 51, 399 (2003).

2. A.V. Sarantsev et al., Phys. Lett. B 659, 94 (2008).

3. U. Thoma et al., Phys. Lett. B 659, 87 (2008).

4. F. Zehr et al., Eur. Phys. J. A 48, 98 (2012).

5. V. Kashevarov et al., Phys. Rev. C 85, 064610 (2012).

6. J. Ajaka et al., Phys. Rev. Lett. 100, 052003 (2008).

7. I. Horn et al., Phys. Rev. Lett. 101, 202002 (2008).

8. I. Horn et al., Eur. Phys. J. A 38, 173 (2008).

9. V. Kashevarov et al., Eur. Phys. J. A 42, 141 (2009).

10. V. Kashevarov et al., Phys. Lett. B 693, 551 (2010).

11. W. Roberts and T. Oed, Phys. Rev. C 71, 055201 (2005).

12. H. Herminghaus et al., IEEE Trans. on Nucl. Science. 30, 3274 (1983).

13. K.-H. Kaiser et al., Nucl. Instr. Meth. A 593, 159 (2008).

14. I. Anthony et al., Nucl. Instr. Meth. A 301, 230 (1991).

15. S.J. Hall, G.J. Miller, R. Beck, P.Jennewein, Nucl. Inst.Meth. A 368, 698 (1996).

16. J.C. McGeorge et al., Eur. Phys. J. A 37, 129 (2008)..

17. A. Starostin et al., Phys. Rev. C 64, 055205 (2001).

18. R. Novotny, IEEE Trans. on Nucl. Science 38, 379 (1991).

19. A.R. Gabler et al., Nucl. Instr. Meth. A 346, 168 (1994).

20. S. Schumann et al., Eur. Phys. J. A 43269 (2010).

21. D. Watts, in Calorimetry in Particle Physics, Proceedings of the 11th International Conference, Perugia, Italy 2004, edited by C. Cecchi, P. Cenci, P. Lubrano, and M. Pepe (World Scientific, Singapore, 2005), p. 560

22. A. Fix and H. Ahrenhövel, Eur. Phys. J. A 25115 (2005).

23. L. Roca, Nucl. Phys. A 748, 192 (2005).

24. M. Oberle et al., Phys. Lett. B 721, 237 (2012).

25. D. Krambrich et al., Phys. Rev. Lett. 103, 052002 (2009).

26. A.V. Anisovich et al., Eur. Phys. J. A 4815 (2012). 